\title{
Références bibliographiques : les langues d'enseignement, un enjeu politique
}

Bernadette Plumelle

\section{OpenEdition}

1 Journals

Édition électronique

URL : https://journals.openedition.org/ries/4524

DOI : $10.4000 /$ ries.4524

ISSN : 2261-4265

Éditeur

France Education international

\section{Édition imprimée}

Date de publication : 1 décembre 2015

Pagination : 159-170

ISSN : 1254-4590

\section{Référence électronique}

Bernadette Plumelle, «Références bibliographiques : les langues d'enseignement, un enjeu politique », Revue internationale d'éducation de Sèvres [En ligne], 70 I décembre 2015, mis en ligne le 18 janvier 2015, consulté le 01 juillet 2021. URL : http://journals.openedition.org/ries/4524 ; DOI : https://doi.org/ $10.4000 /$ ries.4524 


\title{
Références bibliographiques : les langues d'enseignement, un enjeu politique
}

\author{
Bernadette Plumelle \\ Centre de ressources et d'ingénierie \\ documentaires (CRID), CIEP
}

Le choix de la langue par laquelle on enseigne est une question qui se pose avec acuité dans toutes les sociétés. Les aspects politiques, historiques, économiques, sociaux, culturels qui entrent en jeu dans le choix de la langue d'enseignement ont fait l'objet ces dernières années d'une profusion de publications.

Cette bibliographie, non exhaustive, offre un aperçu de la variété des situations éducatives et linguistiques selon les zones géographiques. La première partie donne le cadre général de la situation linguistique dans le monde avec deux éclairages, l'un sur le poids économique des langues, l'autre sur la question des minorités linguistiques. La deuxième partie aborde les politiques éducatives et linguistiques menées dans les pays, en mentionnant les préconisations émises par les organisations internationales. Enfin, la dernière partie propose de nombreuses études de cas nationales qui analysent différentes formes d'enseignement bilingue. Nous avons privilégié des publications très récentes et celles produites dans un contexte francophone mais cette sélection n'épuise pas le sujet ${ }^{1}$. Par ailleurs, on peut se référer à un numéro antérieur de la Revue internationale d'éducation de Sèvres, "l'école et la diversité des cultures " ( $\mathrm{n}^{\circ} 63$, septembre $2013^{2}$ ) qui prend en compte la dimension linguistique à l'école dans des contextes multiculturels.

Sélection terminée le 15 novembre 2015.

\section{UNE SITUATION GÉNÉRALE DE PLURILINGUISME DANS LE MONDE}

\begin{abstract}
CALVET Louis-Jean, CALVET Alain, Les confettis de Babel : diversité linguistique et politique des langues, Éditions Écriture/Paris, OIF/Paris, 2013, 200 p.

L'histoire politique des langues peut être envisagée selon que le droit à la langue, même locale et minoritaire, est imprescriptible ou bien que les "grandes" langues sont incontournables. Cependant les technologies de l'information et de la communication offrent de nouvelles possibilités de diffusion aux différentes langues. Qu'est-ce que la diversité linguistique ? Comment évalue-t-on le "poids» d'une langue? Qu'est-ce qu'une langue menacée ? Après
\end{abstract}

\footnotetext{
1. La bibliothèque du CIEP comprend un fonds documentaire spécialisé dans les politiques éducatives et linguistiques. Le catalogue bibliographique (consultable sur Internet : [http://goo.gl/bBnbqq]) comporte plusieurs milliers de références sur ces sujets. Ainsi, on trouvera de nombreuses références sur la question des langues dans l'enseignement supérieur, la diversification de l'offre de formation linguistique, les stratégies familiales ou la question des langues minoritaires ou régionales.
}

2. Coordonné par A. Akkari. 
avoir présenté la situation linguistique du monde et analysé le poids des différentes langues et les politiques linguistiques menées, les auteurs abordent la situation des langues en danger et la diversité linguistique.

CANUT Cécile, DUCHENE Alexandre (sous la direction de), "Appropriation politique et économique des langues ", Langage et société, juin 2011, n 136, 162 p., bibliogr. Le dossier a pour objet de mettre en évidence la manière dont les langues s'inscrivent dans une série de processus sociaux, politiques et économiques qui font de cet objet un instrument stratégique. La valorisation du plurilinguisme, même si on la retrouve dès le XIX ${ }^{\mathrm{e}}$ siècle, s'inscrit désormais dans un contexte d'économies mondialisées. Les contributeurs, avec des approches variées et à partir de terrains divers (Canada, Hongrie, Suisse, Bulgarie, etc.) portent un regard critique sur l'impact des transformations sociales, politiques et économiques sur les idéologies langagières et sur le rôle du langage et du plurilinguisme dans les sociétés actuelles.

\section{MEISSNER Franz-Joseph coord., "Pluralités, identités, plurilinguismes », Synergies} pays germanophones, $n^{\circ} 4,2011$, p. 3-156

Les pluralités, identités et plurilinguismes sont l'expression de sociétés situées entre mondialisation, "glocalisation » et multilinguismes. Des articles montrent les rapports complexes entre langues traditionnellement dominantes et langues dominées à l'intérieur d'un espace donné (Pérou). D'autres articles s'intéressent à la situation langagière des jeunes issus de l'immigration (Allemagne, Luxembourg) ou en situation d'enseignement bilingue dans une région frontalière (France). [http://goo.gliyTPY9]

\section{Le poids économique des langues}

ALEN GARABATO Carmen, BOYER Henri (sous la direction de), "Regards sur le post-colonialisme linguistique ", Mots : les langages du politique, novembre 2014, $n^{\circ} 106,131 p$.

L'existence de liens privilégiés entre langue et pouvoir n'est plus à démontrer. Ce dossier s'inscrit dans le cadre d'une réflexion continue qui embrasse une grande diversité d'ensembles géopolitiques. L'attention est focalisée sur l'Afrique francophone et l'Amérique hispanophone actuelles.

\section{ATTALI Jacques, La francophonie et la francophilie, moteurs de croissance durable, La Documentation française/Paris, août 2014, 246 p.}

Avec plus de 220 millions de personnes parlant français dans le monde, la francophonie est le sixième espace géopolitique par sa population et peut devenir le quatrième à l'horizon 2050. Selon ce rapport, le potentiel économique de la francophonie est insuffisamment exploité. Parmi les propositions émises pour y remédier, il est préconisé d'augmenter l'offre d'enseignement du et en français, en France et partout dans le monde et de renforcer et étendre l'aire culturelle francophone. [http://goo.gljliaeF]

\section{DUCHENE Alexandre, HELLER Monica, Language in late capitalism: pride and profit, Routledge/New York, 2012, 270 p.}

L'ouvrage s'intéresse à la façon dont nos idées sur la langue et l'identité, habituellement conçues en termes nationaux et politiques comme des questions de droits et de citoyenneté, sont de plus en plus souvent reformulées en termes économiques comme une question de valeur ajoutée. Pour les auteurs, ce changement de discours est lié aux caractéristiques spécifiques de la nouvelle économie mondialisée. En s'appuyant sur dix études de cas, ils démontrent les façons complexes dont les idéologies nationalistes plus anciennes, qui investissent les langues en tant que source de fierté, s'associent à des idéologies néolibérales plus récentes, qui investissent les langues en tant que sources de profit. 
HELLER Monica, "Du français comme "droit" au français comme "valeur ajoutée" : de la politique à l'économique au Canada ", Langage et société, juin 2011, $n^{\circ} 136$, p. 13-30

Le discours sur la langue a beaucoup évolué au Canada. Les revendications nationalistes québécoises ont fait du bilinguisme anglais-français une valeur centrale associée aux droits et aux obligations de la citoyenneté canadienne à partir des années 1960. Le français est traité comme un droit collectif. Ce discours existe toujours, surtout au sein des organismes étatiques québécois, mais à partir des années 1990, il est remplacé, autant dans le discours gouvernemental fédéral, dans le milieu associatif de la francophonie des autres provinces canadiennes, qu'au sein d'une partie de la population québécoise, par un discours insistant sur le poids économique du marché francophone.

\section{RICENTO Thomas éd., Language policy and political economy: English in a global context, Oxford University Press/New-York, 2015, 313 p.}

L'ouvrage explore un nouveau cadre pour la recherche en politique linguistique, qui articule les liens entre politique de la langue et économie politique. L'accent est mis sur l'anglais, une langue qui a joui d'une réputation de «langue mondiale " au cours du XXe siècle et qui est perçue comme un outil de mobilité socio-économique. Cette nouvelle orientation des études en politique linguistique permet d'expliquer pourquoi l'anglais a été vécu comme une bénédiction ou une malédiction selon les parties du monde, pourquoi l'anglais continue d'être utile comme lingua franca dans certains secteurs de l'économie mondiale, et pourquoi il est préjudiciable au développement économique de nombreux pays à faible revenu.

\section{VAN PARIJS Philippe, Linguistic justice for Europe and for the world, Oxford University press, 2011, 299 p.}

En Europe et dans le monde, la connaissance de l'anglais se répand à une vitesse jamais atteinte par aucune autre langue dans l'histoire. Cette domination apparemment irrésistible de l'anglais est souvent perçue et dénoncée comme une injustice flagrante, en donnant un avantage de fait aux locuteurs natifs de langue anglaise. Au contraire, l'auteur, économiste et philosophe, considère que la diffusion de l'anglais au titre de lingua franca doit être encouragée parce qu'elle permet la diffusion de compétences dans une langue véhiculaire commune et, plus fondamentalement, parce qu'elle permet la lutte pour plus de justice en Europe et dans le monde avec une arme essentielle : un moyen peu onéreux de communication et de mobilisation. Cependant, l'usage de l'anglais comme lingua franca s'accompagne d'injustices contre lesquelles il faut lutter activement.

\section{Les minorités linguistiques}

\section{ALEN GARABATO Carmen éd., Gestion des minorités linguistiques dans l'Europe $\boldsymbol{d u} \mathbf{X X I}^{\boldsymbol{e}}$ siècle, Lambert-Lucas/Paris, 2013, 319 p., bibliogr.}

Malgré les efforts uniformisateurs de la plupart des États, de nombreuses minorités linguistiques continuent d'exister sur le vieux continent. Les langues sont de plus en plus considérées comme des éléments du patrimoine. Des instruments de protection à visée universaliste ont été élaborés au sein du Conseil de l'Europe : la Charte européenne des langues régionales ou minoritaires et la Convention-cadre pour la protection des minorités nationales. Mais tous les États n'ont pas signé et ratifié ces traités et la question des minorités continue d'être source de conflits.

CHARBONNEAU François, CERQUIGLINI Bernard, CORREA Sylvio Marcus, et al., "Minorités linguistiques et francophonies en perspective », Glottopol: revue de sociolinguistique en ligne, octobre 2010, $n^{\circ} 16,116 p$.

Ce numéro reprend une partie des actes du colloque «Vues d'ici et d'ailleurs, minorités linguistiques et francophonies en perspective » (Québec, mai 2008), qui a réuni près d'une soixantaine de chercheurs sur les singularités et les points de convergence en matière d'aménagements 
linguistiques. Plusieurs points sont abordés, dont l'intérêt de l'approche comparative dans la question des minorités linguistiques ou la représentation politique des minorités linguistiques (analyse comparée des communautés francophone d'Italie et germanophone de Belgique).

\title{
Politiques LINGUISTIQUES ET ÉDUCATIVES
}

\author{
BEACCO Jean-Claude, BYRAM Michael, Guide pour l'élaboration des politiques \\ linguistiques éducatives en Europe : de la diversité linguistique à l'éducation \\ plurilingue, Conseil de l'Europe/Strasbourg, 2007, 131 p.
}

La finalité de ce guide est de proposer une analyse qui serve de cadre à l'élaboration des enseignements de langues dans les États membres et, à travers lui, à une réflexion sur les politiques linguistiques européennes. La première partie décrit les orientations générales des politiques linguistiques et éducatives en Europe ainsi que les formes d'enseignement des langues qui en découlent. La deuxième partie apporte les données et méthodes nécessaires pour l'élaboration de politiques linguistiques. Enfin, la dernière partie indique les formes d'organisation des enseignements plurilingues. [http://goo.g/8ThTlk]

BONNET-FALANDRY Françoise, DURRANS Stéphanie, JONES Moya (sous la direction de), (Se) construire dans l'interlangue : perspectives transatlantiques sur le multilinguisme, Presses universitaires du Septentrion/Villeneuve d'Ascq, 2015, 249 p.

162 L'interlangue, comme il est rappelé dans l'introduction, «se définit comme la capacité permanente d'individus bi- ou plurilingues à fonctionner entre les langues et les modes de pensée qui les accompagnent ». Ce phénomène linguistique joue un rôle essentiel dans les dynamiques de construction identitaire et nationale et les questions d'interlangue et de multilinguisme sont aujourd'hui au cœur des problématiques d'apprentissage/enseignement dans la plupart des pays du monde.

COSTE Daniel (sous la direction de), Les langues au cour de l'éducation. Principes, pratiques, propositions, E.M.E. \& InterCommunications/Bruxelles, 2013, $285 p$.

Cet ouvrage vise à montrer que l'enseignement des langues et en langues est une dimension centrale de tout système éducatif. Indispensable pour l'accès aux connaissances, pour le développement des compétences et l'ouverture à la diversité culturelle, pour la construction identitaire des jeunes. Il ne semble pas que cette dimension soit pleinement prise en compte dans les curriculums ou les activités et dispositifs didactiques. À partir de la situation française mais aussi de l'expérience d'autres contextes, les auteurs dégagent les voies d'une éducation langagière plus intégrée, cohérente, explicite et inclusive dans le cadre des enseignements et des dispositifs institutionnels existants.

DEPREZ Christine, COLLET Beate, VARRO Gabrielle (sous la direction de), «Familles plurilingues dans le monde : mixités conjugales et transmission des langues : dossier ", Langage et société, mars 2014, $n^{\circ} 147$, p. 7-109

Ce numéro explore, à travers des entretiens, la diversité des pratiques langagières et la créativité des parents et des enfants, donnant à voir des situations très différentes. Le dossier permet de comparer des expériences singulières ou collectives, qui mettent en relief les différents statuts des langues en contact ainsi que le caractère monolingue ou plurilingue de l'environnement social et éducatif. 
FILHON Alexandra, Langues d'ici et d'ailleurs, Cahiers de l'INED, 2009, $n^{\circ} 163$, $288 \mathrm{p}$.

L'ouvrage, dont l'approche est pluridisciplinaire, relate les trajectoires des migrants et de leurs enfants, de leur pays de naissance en Algérie, au Maroc ou en Tunisie à la construction de leur vie en France. Après une première partie qui décrit les contextes linguistiques des trois pays et les langues natales des migrants, l'auteure s'intéresse aux mécanismes de transmission des langues parentales et aux conditions de leur maintien d'une génération à l'autre. Si le poids de certains déterminants sociaux explique la transmission ou l'absence de transmission dans la sphère familiale des langues arabe, berbère et française, les individus peuvent avoir des stratégies anticipatrices pour l'avenir de leurs enfants; c'est l'objet de la troisième partie, avec le cas des couples dits « mixtes».

MAURER Bruno (sous la direction de), "Les langues des apprenants dans les systèmes éducatifs post-coloniaux", Glottopol: revue de sociolinguistique en ligne, juillet 2013, $n^{\circ} 22,228 p$.

Depuis le milieu des années 1990, l'éducation pour tous a entraîné une massification de l'enseignement, accompagnée d'une ruralisation. L'arrivée en nombre de ruraux, moins exposés que les urbains aux langues officielles, pose la question des langues d'enseignement. Aujourd'hui, l'accent est mis sur leur partenariat et conduit à interroger le lien entre langue(s) de scolarisation et qualité de l'enseignement, particulièrement pour l'enseignement primaire. Comment réduire l'échec scolaire massif faute d'une maîtrise suffisante des langues d'enseignement ? Quelle place accorder aux langues nationales dans l'enseignement ? Les contributions permettent d'aborder des contextes différents : la zone créolophone (île de La Réunion, île Maurice, Jamaïque); le monde arabe (Algérie) ; l'Afrique subsaharienne (Sénégal, Cameroun, Tchad, Madagascar); la région Pacifique (plurilinguisme éducatif au Vanuatu). [http://goo.g//acFzXI]

PULINX Reinhilde, VAN AVERMAET Pie, BUTTARO Lucia, et al., "La langue facteur d'intégration et d'insertion ", Revue française de linguistique appliquée, décembre 2014, vol. XIX-2, 119 p., bibliogr.

Avec le développement des mobilités, la maitrise de la langue devient un facteur clé dans la réussite de l'intégration et de l'insertion sociale et professionnelle. La question de la maîtrise de la langue en contexte d'intégration et d'insertion fait l'objet de ce numéro. Les articles présentent des travaux et des approches variées, en termes de contextes géographiques (Belgique, États-Unis, France, Suisse) comme de publics. [http://goo.gl/Kwio1w]

\section{RAMANATHAN Vaidehi éd., Language policies and (dis)citizenship: rights access pedagogies, Multilingual Matters/Bristol, 2013, 297 p.}

L'ouvrage explore le concept de citoyenneté et fait valoir qu'il devrait être compris à la fois comme un processus et comme la capacité à participer pleinement. Les auteurs utilisent des contextes locaux pour mettre en avant la façon dont les personnes vulnérables, en particulier celles issues de milieux linguistiques minoritaires, continuent d'être exclues, tout en offrant une démonstration du potentiel de changement existant. Les contributions abordent des thèmes tels que le rôle joué par les politiques linguistiques et les pratiques pédagogiques.

TOLLEFSON James W., "Language Policy-Making in Multilingual Education: Mass Media and the Framing of Medium of Instruction ", Current Issues in Language Planning, 2015, vol. $16 n^{\circ} 1-2$, p. 132-148

Dans certains contextes multilingues, les politiques des langues d'enseignement dans l'éducation font l'objet de débats publics dans les médias, sur des questions telles que l'identité culturelle nationale, la sécurité nationale, l'équité et la justice sociale. Comment les médias de masse façonnent-ils les politiques adoptées dans des contextes multilingues? Cet article présente une 
analyse comparative de deux controverses dans les principaux journaux des pays concernés : la langue maternelle versus l'anglais comme langue d'enseignement à Hong Kong et le débat sur le rôle de l'anglais et de l'espagnol en Arizona.

\section{WEBER Jean-Jacques, Flexible multilingual education: putting children's needs} first, Multilingual Matter/Bristol, 2014, 218 p.

L'ouvrage préconise l'éducation multilingue flexible, qui se fonde sur les ressources réelles de l'enfant à la maison et donne accès aux langues locales mais aussi mondiales dont les élèves ont besoin pour leur réussite scolaire et professionnelle. Dans la mesure où, dans un monde globalisé, un nombre croissant d'enfants grandit en contexte multilingue, le besoin se fait sentir de solutions multilingues plus diversifiées au sein des politiques linguistiques éducatives. Les études de cas révèlent que l'éducation multilingue souple, plutôt que l'éducation en langue maternelle, est le moyen le plus prometteur de se rapprocher de l'objectif de l'équité en éducation.

\section{Préconisations des organisations internationales}

Conseil de l'Europe, L'importance de compétences en langue(s) de scolarisation pour l'équité et la qualité en éducation et pour la réussite scolaire : recommandation CM/REC (2014) 5 adoptée par le Comité des Ministres du Conseil de l'Europe et exposé des motifs, Conseil de l'Europe/Strasbourg, 2014, 24 p.

Le manque de maîtrise de formes linguistiques diversifiées détermine en partie l'échec scolaire, comme l'a montré l'enquête PISA de l'OCDE. La maîtrise de la ou des langue(s) de scolarisation représente l'une des voies devant permettre d'atteindre l'objectif - que se fixent la Commission européenne et de nombreuses autorités au niveau national - d'une réduction du nombre d'élèves quittant le système scolaire sans qualification. Les mesures en faveur d'une maîtrise suffisante des compétences en langue de scolarisation par tous les élèves relèvent de la lutte contre l'échec scolaire et participent ainsi au premier chef à la recherche d'équité et de qualité dans l'éducation. [https://goo.gl/eD7uer]

\section{Conseil de l'Union européenne, Conclusions du Conseil sur le plurilinguisme et le développement des compétences linguistiques, Conseil de l'Union européennel Bruxelles, 20 mai 2014.}

Les membres du Conseil de l'Union européenne ont adopté des conclusions en faveur d'un renforcement des mesures destinées à promouvoir le plurilinguisme et à améliorer la qualité et l'efficacité de l'apprentissage et de l'enseignement des langues. La diversité linguistique est considérée comme une « composante essentielle de la culture européenne et du dialogue interculturel » et le multilinguisme est une compétence clé qui favorise « la mobilité, l'employabilité et l'épanouissement personnel des citoyens européens ». [http://goo.gl/SsPpxh]

INGLIS Christine, Planning for cultural diversity, UNESCO. IIPE/Paris, 2008, $181 \mathrm{p}$.

Garantir l'égalité en matière d'éducation, garantir les droits au maintien des cultures et à la pleine et entière participation des groupes minoritaires à la vie de la société, tout en préservant l'harmonie sociale et le développement national au sein d'une société démocratique, est un défi majeur. Cet ouvrage évoque plusieurs processus permettant d'élaborer des réponses adaptées à la diversité culturelle. Trois grands axes de stratégie sont étudiés : l'organisation et les structures de l'éducation; les programmes scolaires, la pédagogie et le choix de la langue; les relations entre l'école et la communauté. [http://g00.gl/a8kpl6] 
UNESCO. Bureau régional pour l'éducation en Asie et dans le Pacifique, Why language matters for the Millennium Development Goals, UNESCO/Bangkok, 2012, 54 p.

Cette publication s'appuie sur les présentations de la conférence «International Conference on Language, Education and the Millennium Development Goals» (Bangkok, novembre 2010). La langue est au centre de l'activité humaine, de l'expression de soi et de l'identité. Les participants à cette conférence, convaincus que la langue est un outil essentiel pour la réalisation des Objectifs du millénaire pour le développement (OMD), ont relaté les nombreuses façons dont les initiatives visant à promouvoir les langues locales ont un réel impact dans la vie des gens et ont montré en quoi l'éducation précoce dans la langue maternelle améliore la vie des enfants et de leurs communautés. [http://goo.g//yHEePR]

UNESCO : Institut de l'UNESCO pour l'apprentissage tout au long de la vie, ADEA : Association pour le développement de l'éducation en Afrique, Pourquoi et comment l'Afrique doit investir dans les langues africaines et l'enseignement multilingue : note de sensibilisation et d'orientation étayée par les faits et fondée sur la pratique, Hambourg: UIL-UNESCO, Paris : ADEA, 2010, 71 p.

Dans une publication de 1953, l'Unesco a souligné l'importance d'éduquer les enfants dans leur langue maternelle. Plus de 50 ans après, la plupart des pays africains continuent d'utiliser la langue de l'ex-pays colonisateur comme principale langue d'enseignement et de gouvernement du pays. Cette note de sensibilisation a pour objectif de souligner le rôle pivot des langues dans un apprentissage de qualité. Elle vise en particulier à dissiper les préjugés et la confusion qui règnent à propos des langues africaines. Cette note met à profit les recherches existantes et pratiques en cours pour débattre de la politique linguistique la mieux adaptée aux besoins de l'Afrique en matière d'éducation. http://goo.g/BYTaNj

\section{LANGUES D'ENSEIGNEMENT ET CONTEXTES MULTILINGUES : ÉTUDES DE CAS}

BAHOUS Rima, BACHA Nahla Nola, " Multilingual educational trends and practices in Lebanon: a case study », International review of education, novembre 2011, vol. 57, $n^{\circ}$ 5-6, p. 737-749

Cet article présente le contexte multilingue du Liban ainsi que ses politiques et pratiques linguistiques dans l'éducation. Les auteures analysent plus spécifiquement dans quelle mesure la nature multilingue du pays se reflète dans les politiques linguistiques de l'enseignement. Les résultats montrent que de nombreux efforts ont été déployés pour introduire une langue d'enseignement et une troisième langue, selon une décision du ministère de l'éducation, tout en cultivant l'arabe, langue nationale.

BOIDIN Capucine, "Le double discours des politiques d'éducation interculturelle bilingue au Paraguay ", Problèmes d'Amérique latine, $n^{\circ}$ 92, 2014/2, p. 73-90

La nouvelle Constitution du Paraguay a reconnu en 1992 le guarani comme langue officielle sur tout le territoire national aux côtés de l'espagnol. Depuis 1994 une politique nationale d'éducation bilingue interculturelle a été lancée. Le Paraguay est proclamé comme étant un pays "bilingue et pluriculturel ». Si, au niveau politique, le guarani bénéficie d'une forte reconnaissance symbolique, au niveau de la planification linguistique, de nombreuses résistances envers l'usage officiel du guarani peuvent être observées. 
GARNIER Bruno coord., "Insécurité linguistique en éducation : approche sociologique comparée des élèves issus de Maghreb ", Études de linguistique appliquée, septembre 2014, $n^{\circ} 175$, p. 261-369

Ce numéro est issu d'un symposium intitulé « Multiculturalisme et insécurité linguistique en éducation dans l'espace méditerranéen; approche sociologique comparée des élèves issus du Maghreb» (Rabat, 2012). L'article introductif aborde les conditions d'adaptation et d'acclimatation du français à différents milieux, avant de s'intéresser à son implantation au Maghreb. Les contributions suivantes abordent les situations algérienne et marocaine, dont l'introduction de la langue amazighe dans le système éducatif.

\section{LAROUSSI Foued, LIENARD Fabien (sous la direction de), Plurilinguisme poli-} tique, linguistique et éducation : quels éclairages pour Mayotte ?, Publications des universités de Rouen et du Havre/Rouen, 2011, 494 p.

Sur le territoire de Mayotte, île française de l'océan Indien, deux langues locales, l'une d'origine bantoue, le shimaore, l'autre d'origine malgache, le kibushi, cohabitent avec la langue officielle et de scolarisation, le français. Ce volume, en rendant compte aussi d'expériences et de travaux qui ont été menés dans d'autres pays ou dans des régions dont les situations linguistiques ressemblent à celle de Mayotte, éclaire la situation linguistique mahoraise et contribue à approfondir le débat sur les rapports entre plurilinguisme, politique linguistique et éducation.

NOCUS Isabelle, VERNAUDON Jacques, PAJA Mirose (sous la direction de), L'école plurilingue en outre-mer : apprendre plusieurs langues pour apprendre, Presses universitaires de Rennes/Rennes, 2014, 505 p.

L'ouvrage dresse un état des lieux de la recherche française sur le plurilinguisme précoce à l'école et sur la prise en compte des langues maternelles ou d'origine des élèves dans les collectivités françaises d'outre-mer (Nouvelle-Calédonie, Polynésie française et Guyane), dans un champ encore largement dominé par les perspectives anglophones nord-américaines. Il propose une comparaison entre trois collectivités dont les citoyens « autochtones » ont vu certaines de leurs revendications scolaires, linguistiques et culturelles, prises en compte à l'école depuis quelques années.

OMER Danielle, TURPIN Frédéric (sous la direction de), Éducations plurilingues : l'aire francophone entre héritages et innovations, Rennes: Presses universitaires de Rennes, 2013, 236 p.

L'ouvrage rassemble des textes sur des contextes éducatifs plurilingues dans une perspective francophone. Les études portent sur un contexte où le français est une langue présente dès l'école primaire comme langue d'enseignement ou comme langue de scolarisation dans des pays (ou régions) où le français est une langue seconde pour une partie importante de la population. L'axe central des contributions est celui de la situation linguistique des publics scolaires dont le répertoire linguistique premier n'est pas introduit comme langue d'enseignement à l'école.

\section{Études de cas en Afrique}

ALTMAYER Claus, WOLFF H. Ekkehard (ed.), Africa: challenges of multilingualism = Afrika: herausforderungen der mehrsprachigkeit $=$ les défis du plurilinguisme en Afrique, Peter Lang/Frankfurt am Main, 2013, 252 p.

L'ouvrage rassemble des communications présentées entre 2009 et 2012 au sein du réseau GANAA (german-african network of alumni and alumnae). Les contributions couvrent une grande variété de situations culturelles, politiques et éducatives du multilinguisme en Afrique. Après un article introductif sur la situation linguistique en Afrique, les auteurs, chercheurs africains, présentent des situations sociolinguistiques principalement au Bénin, au Kenya, au 
Mali, au Sénégal, en Afrique du Sud, au Soudan et au Togo. Ils portent sur différents aspects comme l'autonomisation de la langue et de la protection de la diversité linguistique ou la question des langues, de l'enseignement primaire à l'enseignement supérieur.

BROCK-UTNE Birgit, MERCER Malcom, «Using African languages for democracy and lifelong learning in Africa: a post-2015 challenge and the work of CASAS », International review of education, décembre 2014, vol. 60, $n^{\circ} 6, p .777-792$

Les Africains utilisent dans leur quotidien leurs langues locales, alors que l'enseignement dans les établissements scolaires est dispensé dans une langue exogène. Dans de nombreux cas, l'éducation des adultes est réalisée dans une langue que la majorité des apprenants ne parlent pas. Un argument souvent avancé contre l'usage des langues africaines dans l'instruction est qu'elles existent en très grand nombre et qu'il serait difficile d'en choisir une comme langue d'enseignement. Mais est-ce vraiment le cas ? Et est-il nécessaire de sélectionner une seule langue? La mission principale du Centre d'études avancées sur les sociétés africaines (Centre for Advanced Studies of African Society, CASAS) basé au Cap consiste à harmoniser les formes écrites de la majorité des langues africaines, afin de pouvoir les utiliser comme langues d'instruction, des gouvernements et de la presse. [http://goo.g/0808RY]

Centre international d'études pédagogiques, Réussir l'éducation en Afrique: l'enjeu des langues - CIEP, 27-28 mars 2014. Synthèse de la conférence internationale, CIEP/Sèvres, mai 2015, 16 p.

Ce document propose une synthèse des discours d'ouverture, de la conférence inaugurale et des tables rondes de la conférence internationale organisée par le CIEP en partenariat avec le ministère des Affaires étrangères, l'Agence française de développement, des organisations internationales (AUF, ADEA, CONFEMEN, OIF, PME) et la direction du développement et de la coopération de la Confédération suisse. Des exemples de politiques linguistiques ont été donnés et plusieurs interventions ont porté sur la mesure des acquis de l'apprentissage, la formation des enseignants ou le financement de programmes éducatifs en contexte multilingue. [http://g0o.g/FMrEe8]

DIABATE Arouna, "L'enseignement du français en contexte multilingue au Burkina Faso : problématique de la généralisation de l'expérimentation de l'éducation bilingue dans l'enseignement de base ", Travaux de didactique $\boldsymbol{d u}$ français langue étrangère, 2010, $n^{\circ}$ 63, p. 169-118

Le Burkina Faso a pour langue officielle le français et compte environ soixante langues nationales. L'éducation bilingue est une innovation pédagogique conçue par des chercheurs et des praticiens de l'éducation burkinabé, avec l'appui technique et financier d'une ONG suisse. L'auteure donne la définition de l'éducation bilingue en contexte burkinabé et décrit le dispositif au niveau préprimaire, à l'école primaire et au collège. Elle met en lumière les obstacles (réticence de la population, hésitation de l'État, évaluation des apprentissages) et donne quelques perspectives.

GUIDI Pierre, «Les enfants du Wolaitta n'apprendront pas en wogagoda : les enjeux linguistiques et politiques d'un conflit scolaire en Éthiopie (1999-2000) ", Cahiers de la recherche sur l'éducation et les savoirs, $n^{\circ} 11,2012$, p. 129-148

En 1999-2000, une révolte a éclaté dans le Wolaitta, région du Sud éthiopien, à propos de la langue d'enseignement. Menée par les enseignants, les élèves et des notables importants, elle a rapidement gagné l'ensemble de la population, sans distinction de classe. Après six années d'éducation dans la langue locale, le wolaittigna, le gouvernement a décidé d'introduire le wogagoda, un "espéranto " créé à partir de quatre langues omotiques parlées dans la région. Le but du gouvernement était d'unifier culturellement et politiquement la région pour en faciliter le contrôle et briser l'hégémonie régionale du Wolaitta. L'analyse détaillée de ce conflit scolaire dévoile des enjeux culturels, identitaires et politiques extrêmement lourds, liés à la question linguistique. [http://cres.revues.org/2263] 


\section{MAURER Bruno, Les langues de scolarisation en Afrique francophone : enjeux et repères pour l'action. Études pays, AUF/Paris, juin 2010, 759 p.}

Plusieurs pays d'Afrique ont introduit, ces dernières années, l'enseignement en langues nationales africaines dans le cycle primaire, en complément du français. Ces expérimentations ont été documentées et analysées pour identifier les effets, les difficultés de mise en œuvre et consolider les processus de réforme dans le cadre d'un programme d'étude (LASCOLAF) financé par l'OIF, l'AUF, le ministère français des Affaires étrangères et européennes et l'Agence française de développement. Ce volume comprend cinq études de pays: Bénin, Burundi, Burkina Faso, Cameroun, Niger, Sénégal. Pour chacune, on trouve la politique linguistique menée, la mise en œuvre pédagogique dans l'éducation de base ; la formation des enseignants ; les résultats et impacts des politiques linguistiques et enfin, les recommandations et préconisations. [http://goo.g/SdpCT4]

\section{MUNYANKESHA Pascal, Les défis du plurilinguisme officiel au Rwanda : analyse sociolinguistique, Presses académiques francophones/Allemagne, 2013, 275 p., bibliogr.}

À la différence d'autres pays d'Afrique subsaharienne, le Rwanda se caractérise par une forte homogénéité linguistique. Il existe depuis 1996 un trilinguisme officiel, proclamé par arrêté présidentiel, le kinyarwanda (langue nationale), le français et l'anglais mais il existe des problèmes importants de mise en application de ce trilinguisme. L'auteur, qui a mené une enquête sur les obstacles rencontrés, considère ce trilinguisme comme un défi politique.

ROSEKRANS Kristin, SHERRIS Arieh, CHATRY-KOMAREK Marie, "Education reform for the expansion of mother-tongue education in Ghana ", International review of education, octobre 2012, vol. $58, n^{\circ} 5$, p. 593-618

En 1957, le Ghana était le premier État-nation colonial d'Afrique subsaharienne à obtenir son indépendance de l'autorité britannique. L'anglais est cependant resté la langue d'alphabétisation pendant la majeure partie de ces années d’indépendance; parmi les langues autochtones en usage aujourd'hui, on dénombre 11 langues principales et 67 secondaires. Les auteurs analysent la création d'un cadre de politiques favorable à l'enseignement bilingue, en vue d'appliquer un programme multilingue global et innovant, le programme national d'accélération de l'alphabétisation (National Literacy Acceleration Program, NALAP), déployé dans les écoles du pays au début des années 2010.

RWANTABAGU Hermenegilde, "Tradition, globalisation and language dilemma in education: african options for the 21 st century ", International review of education, septembre 2011, vol. 57, $n^{\circ} 3-4$, p. 457-475

En étudiant des pays africains et le cas du Burundi en particulier, l'auteure analyse les politiques adoptées visant à promouvoir l'usage de la langue maternelle en tant que base pour l'acquisition des connaissances et l'intégration culturelle. Le Burundi a traversé une série de réformes éducatives tant avant qu'après l'accession à son indépendance en 1962, le français et le kirundi se trouvant en concurrence en tant que langues d'enseignement. Après l'intégration du Burundi à la Communauté d'Afrique de l'Est en juillet 2007, l'anglais et le kiswahili ont été ajoutés aux programmes scolaires, compliquant ainsi les politiques éducatives.

WILDSMITH Rosemary, "The African languages in South Africa education 2009-2011 ", Language teaching, janvier 2013, vol. 46, $n^{\circ} 1$, p. 120-124

La politique éducative et linguistique d'Afrique du Sud considère le multilinguisme comme l'un de ses principaux objectifs. La mise en œuvre d'une telle politique est cependant un processus lent, en particulier dans le domaine de l'éducation, où les parents, les enseignants et les élèves favorisent l'anglais, langue dominante pour des raisons à la fois historiques et instrumentales. Ce texte fait état des progrès de la recherche dans la promotion et l'utilisation des langues africaines dans l'éducation en Afrique du Sud au cours des dernières années. 


\section{Études de cas en Europe}

BUSCH Brigitta, "Trends and innovative practices in multilingual education in Europe: an overview ", International review of education, novembre 2011, vol. 57, $n^{\circ}$ 5-6, p. 541-549

Cet article présente un panorama de la situation en Europe. Au cours des cinquante dernières années, l'axe de la recherche est passé d'un intérêt initial pour les identités des locuteurs et pour leur appartenance à des communautés supposées homogènes à une mise en lumière des divers répertoires (multi)linguistiques des apprenants. Par suite des mouvements régionalistes apparus dans les années 1960 et de l'accroissement de la transmigration et de la mobilité mondiale à partir des années 1990 ont été élaborés différents modèles de pratiques et de politiques pour l'enseignement bilingue et multilingue en Europe.

CARTY Nicola, "The adult learner in Gaelic language-in-education policy: language revitalisation and the CEFR", European journal of language policy = Revue européenne de politique linguistique, novembre 2014, vol. 6, n 2, p. 195-217

En Écosse, comme au Royaume-Uni, l'éducation multilingue gagne de l'importance. Une priorité politique supplémentaire en Écosse est la revitalisation du gaélique écossais, après des siècles de déclin. À l'intersection de ces domaines prioritaires se trouve la politique du gaélique comme langue intégrée à l'éducation. Or la politique linguistique éducative en faveur du gaélique ne contribue pas actuellement à la revitalisation de la langue. L'article examine certaines des questions liées à la politique de la langue dans l'éducation, en relation avec les apprenants adultes de gaélique.

GADELII Karl, LOFSTROM Jonas (coordinateurs), "Les langues nordiques à l'ère de la mondialisation : dossier ", Nordiques, $n^{\circ} 24$, novembre 2012, 147 .

Les pays nordiques présentent une variation dialectale importante, notamment en Norvège et en Suède, et hébergent des minorités avec des langues reconnues (pays scandinaves). Ils englobent également des communautés importantes issues d'une immigration récente. Enfin, ils font constamment face à l'influence de l'anglais, particulièrement dans les domaines de la recherche et du monde des affaires. Face à cette situation, ces pays ont ressenti le besoin de formuler une politique linguistique commune et de légiférer sur le statut des langues.

GORTER Durk, CENOZ Jasone, " Multilingual education for European minority languages: the Basque Country and Friesland ", International review of education, vol. 57, $n^{\circ}$ 5-6, novembre 2011, p. 651-666

Le système éducatif de la Communauté autonome du Pays basque a connu une importante transformation amorcée en 1979, alors que moins de $5 \%$ des enseignants étaient en mesure d'exercer en langue basque. Aujourd'hui, ce chiffre est passé à plus de $80 \%$. Une approche innovante a été adoptée pour enseigner la langue minoritaire basque, parallèlement à la langue dominante, l'espagnol, et à la langue internationale, l'anglais. Le résultat est une amélioration considérable de la maitrise du basque parmi les jeunes. En revanche, pour la langue frisonne aux Pays-Bas, les avancées au cours des trente dernières années ont été beaucoup plus lentes et les résultats plus modestes.

HAWKEY James, " Meeting language-in-education policy aims: Catalonia in the twenty-first-century ", European journal of language policy = Revue européenne de politique linguistique, avril 2014, vol. 6, $n^{\circ} 1$, p. 5-21.

L'article porte sur la politique linguistique éducative en Catalogne. L'auteur examine certaines conséquences du changement de langue véhiculaire du système scolaire (du castillan au catalan). La nouvelle langue véhiculaire du système éducatif catalan est née de plusieurs lois et décrets importants, dont la Llei de Normalització Lingüística de 1983, qui avait comme objectif «l'extension de la connaissance du catalan ». Dans quelle mesure cet objectif est-il atteint ? 
SIAROVA Hanna, ESSOMBA Miquel Àngel, Soutien linguistique pour les jeunes issus de l'immigration : les politiques qui soutiennent efficacement l'inclusion, Migration Policy Institute Europe / Washington, juillet 2014, 9 p.

L'objectif de ce document est de donner un aperçu synthétique des politiques de soutien linguistique disponibles en Europe pour les élèves immigrés, et d'identifier les lacunes dans leur mise en application. Il recommande des mesures et des directions à prendre lors de l'élaboration des stratégies linguistiques nationales pour répondre aux besoins des élèves immigrés, depuis les pratiques au niveau de l'école de la langue de l'État et de l'enseignement de la langue maternelle jusqu'à une approche communautaire et la professionalisation de tous les parties concernées. [http://goo.g/ndfkxU] 